\title{
Design and characterization of a fractal-inspired multi-frequency piezoelectric energy converter
}

\author{
Davide Castagnetti \\ Department of Engineering Sciences and Methods, \\ University of Modena and Reggio Emilia, Via Amendola 2, 42122 Reggio Emilia, Italy \\ davide.castagnetti@unimore.it
}

\begin{abstract}
A promising harvesting technique, in terms of simplicity and efficiency, is the conversion of ambient kinetic energy through piezoelectric materials. This work aims to design and investigate a piezoelectric converter conform to a fractal-inspired, multi-frequency structure previously presented by the author. A physical prototype of the converter is built and experimentally examined, up to $120 \mathrm{~Hz}$, in terms of modal response and power output. Three eigenfrequencies are registered and the power output is particularly good at the fundamental eigenfrequency. Also the effect of the resistive load applied to the converter is investigated.
\end{abstract}

KEYWORDS. Energy harvesting; Piezoelectric converter; Multi-frequency structures; Fractal geometry; Power generation.

\section{INTRODUCTION}

7 he study of energy harvesting devices, able to convert ambient energy into electrical energy, is increased, in recent years, together with the development of wireless sensor nodes. The most common source for energy harvesting is kinetic energy, since it is ubiquitous, easily accessible, and present in the form of vibrations or random forces. According to [1], the typical range of ambient vibrations is below $100 \mathrm{~Hz}$, therefore identifying simple structures that efficiently harvest kinetic ambient energy in this range is challenging. Among the available conversion technologies [2-3], piezoelectric materials have the peculiarity of simplicity and high conversion efficiency [2] in the harvesting of ambient kinetic energy. Many piezoelectric energy harvesters have been proposed in the literature [4-11], relying on different architectures. However, a cantilever beam configuration is the most common solution [12-15], for piezoelectric converters since it generates large deflection strains when operated at its fundamental frequency and a desired eigenfrequency can be easily obtained varying its length or introducing an appropriate proof mass [16-18]. By assembling a batch of cantilevers [19-21] each of them tuned at a different fundamental frequency, a multi-frequency converter is obtained. However, the global efficiency is low, since a single cantilever is operated at each resonant frequency.

To overcome this drawback, in [22] the author proposed and computationally examined four fractal-inspired structures, that provide many eigenfrequencies evenly distributed in the range between 0 and $100 \mathrm{~Hz}$ and convert energy more efficiently than a traditional batch of cantilevers. This frequency range was chosen in order to develop structures that efficiently convert ambient vibrations, which are mainly and widely distributed in this range [1]. A subsequent experimental investigation of the two most performing structures [23], confirmed the good modal response. This work aims at investigating a piezoelectric converter inspired to one of these fractal-inspired, multi-frequency structures. The piezoelectric converter is designed and then a physical prototype is experimentally investigated in terms of modal response and power output in the frequency range between 0 and $120 \mathrm{~Hz}$. The converter prototype was made of a support steel plate and thin piezoelectric sheets of commercial PSI-5H4E [24]. Three eigenfrequencies are registered below $120 \mathrm{~Hz}$ and a good power generation is obtained, in particular at the first eigenfrequency. 


\section{METHOD}

\section{Design of the pieqoelectric converter prototype}

7 he piezoelectric converter prototype here proposed relies on the fractal-inspired multi-frequency structure in Fig. 1, which was proposed and examined by the author in [22] and [23]. Fig. 2 shows the sketch of the prototype of the piezoelectric converter, which is obtained by applying to the support plate in Fig. 1 thin rectangular laminas of piezoelectric material (hatched area in Fig. 2) for each inner cantilever. The piezoelectric patches are close to the constrained side of the structure, where maximum stresses (and hence strains) originates in case of vibration induced deflections. The support plate is made of a thin sheet of steel (S235JR) with a thickness of $0.2 \mathrm{~mm}$. This thickness value represents the best trade-off between the need to provide an adequate mechanical strength and to obtain a large number of eigenfrequencies in the frequency range below $120 \mathrm{~Hz}$. The piezoelectric patches are commercial PSI-5H4E [24], with a thickness of $0.267 \mathrm{~mm}$, and were joined to the support plate through a bi-adhesive tape from $3 \mathrm{M}$ which provides an adequate electrical insulation. Moreover, the adhesive tape maximizes the distance of the piezoelectric layer from the neutral plane of the structure, thus increasing strain and electrical generation and is highly compliant, allowing higher deflections of the lamina.
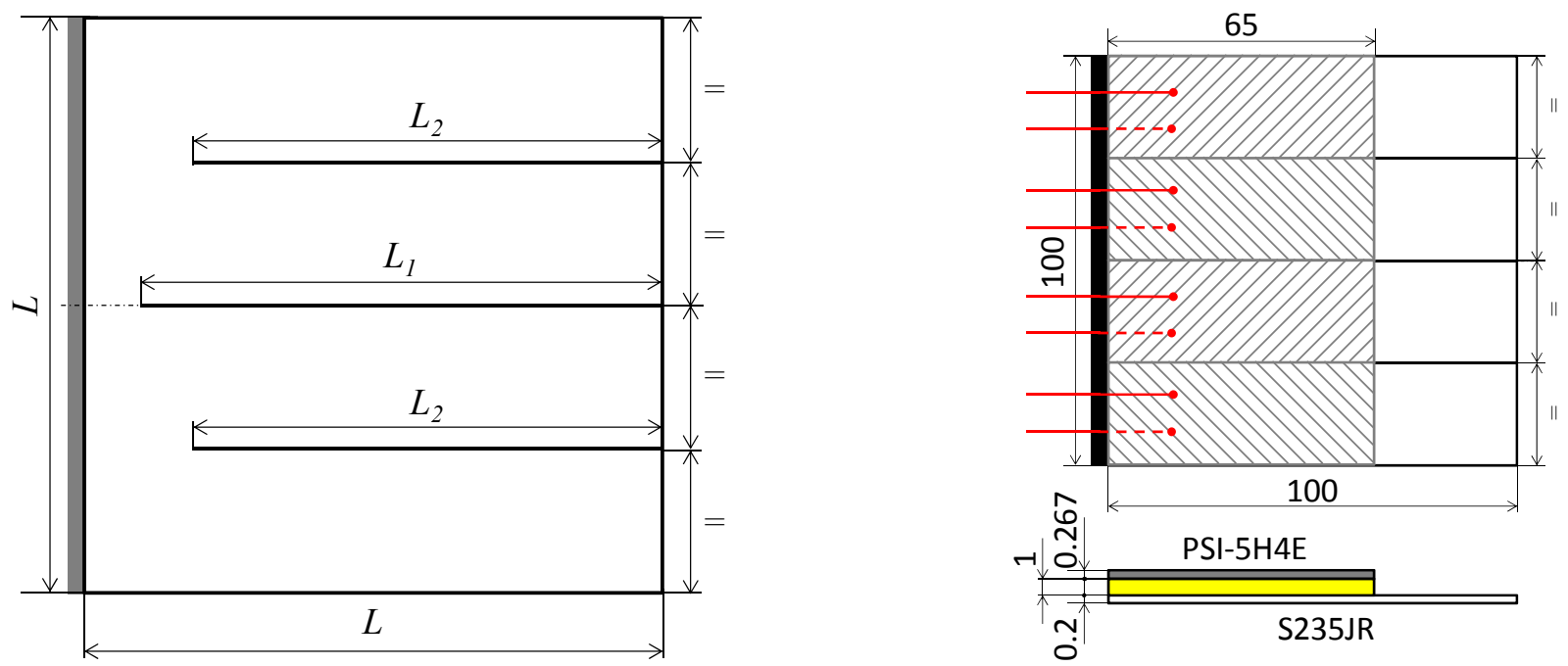

Figure 1: Sketch of the fractal-inspired geometry for the Figure 2: Sketch of the prototype of the piezoelectric converter piezoelectric converter prototype

Tab. 1 collects the mechanical and electrical properties of the piezoelectric patches, used to build the prototype. These piezoelectric patches include nickel electrodes and connecting wires on both sides. The support plate was manufactured through laser-jet cutting.

\begin{tabular}{cc}
\hline Piezoelectric strain coefficient, $d_{31}[\mathrm{~m} / \mathrm{V}]$ & $-320 \times 10^{-12}$ \\
Relative dielectric constant, $k_{3}$ & 3800 \\
Mass density, $\rho\left[\mathrm{kg} / \mathrm{m}^{3}\right]$ & 7800 \\
Young's modulus, $E[\mathrm{GPa}]$ & 62 \\
Poisson's ratio, $v$ & 0.3 \\
Structural damping & 0.02 \\
\hline
\end{tabular}

Table 1: Electrical and mechanical properties of PSI-5H4E

\section{Experimental campaign}

Fig. 3 shows a picture of the converter prototype, built according to the sketch in Fig. 2, and experimentally examined in order to investigate its modal response and the power output between 0 and $120 \mathrm{~Hz}$. Table 2 reports the two variables 
examined in the full factorial experimental campaign. The first variable is the amplitude of the acceleration applied to the converter prototype: $0.5 \mathrm{~g}$ or $1 \mathrm{~g}$, respectively, where $\mathrm{g}$ is the gravitational acceleration. The second variable is the value of electric resistance applied in series to each piezoelectric patch, which varies over three levels: $6.8 \mathrm{M} \Omega, 100 \mathrm{k} \Omega$, and 10 $\mathrm{k} \Omega$. On the one hand, the first very high value allows to simulate the maximum output voltage in a nearly open circuit condition. Despite very high, the resistance avoided that the generated electric charges were stored on the electrodes transforming the piezoelectric patch in a capacitor. On the other hand, the second and third resistance values were chosen to investigate the effect of different resistive loads on the power output of the piezoelectric patch.

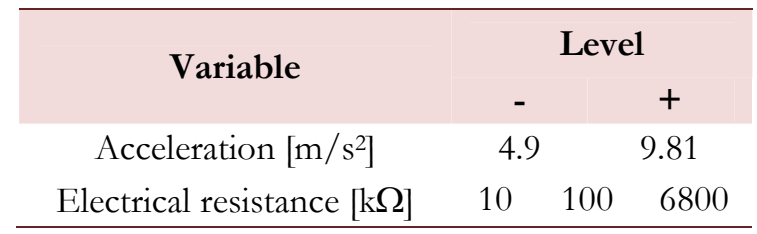

Table 2: Variables of the full factorial experimental plan.

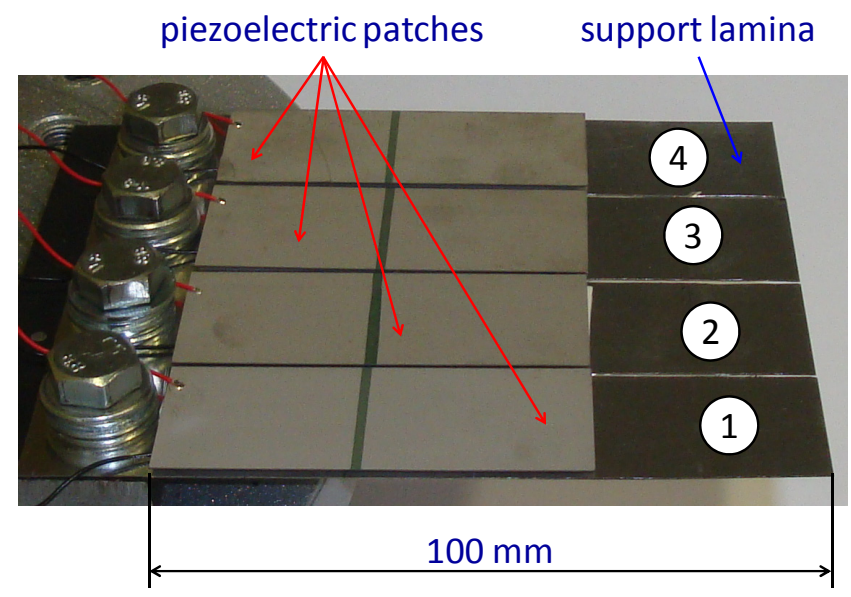

Figure 3: Physical prototype of the fractal-inspired piezoelectric converter.

Through an electro-dynamic shaker (Data Physics BV400 [25]), the converter was stressed by a sinusoidal excitation, whose frequency sweeps in the range from $0 \mathrm{~Hz}$ to $120 \mathrm{~Hz}$. In order to implement a closed-loop control on the system, a miniature accelerometer (MMF KS94B100 [26]) was applied to the vibrating table of the shaker, by fixing it through a magnetic base. The shaker was managed by an 8 channels Abacus controller and the whole testing apparatus was controlled by the Signal Star software, installed on a PC that moreover performs data acquisition. A Polytec point laser Doppler vibrometer, equipped with a OFV-505 sensor head and controlled by a Polytec OFV-5000 controller [27], was used to identify the eigenfrequencies of the converter prototype. The laser vibrometer was set up vertically on the plate, and measured both the speed and the deflection of the tip of the cantilevers during the tests (Fig. 4). A sensitivity of 500 $\mathrm{mm} / \mathrm{s} / \mathrm{V}$ was set to measure the speed, while for the displacement measurement the sensitivity of the vibrometer was set to $5 \mathrm{~mm} / \mathrm{V}$ and $100 \mu \mathrm{m} / \mathrm{V}$ for the first and subsequent eigenfrequencies, respectively. Also the data from the laser Doppler vibrometer were registered through the Signal Star software which controls the shaker.

Each of the four piezoelectric patches on the converter was electrically connected to a 16 channels data acquisition module (USB 6251 [28]). The data acquisition module was connected through a USB port to a notebook equipped with the Labview software [29] and the output voltage of each piezoelectric lamina was registered through the Labview SignalExpress application [29].

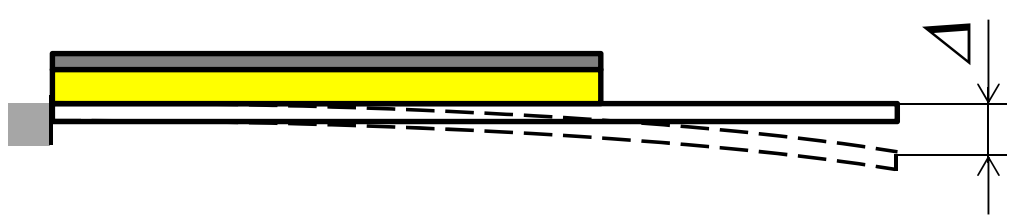

Figure 4: Sketch showing the measurement of the tip deflection, $\Delta$, on the converter. 


\section{RESULTS}

$\mathrm{F}$ Ig. 5 shows the tip speed registered for lamina \#1 (solid line) and \#2 (dashed line), through the laser Doppler vibrometer. In order to assure a good measure also for high deflections occurring at the fundamental eigenfrequency, the speed was measured $5 \mathrm{~mm}$ far from the tip of the lamina. Since the eigenmodes below $120 \mathrm{~Hz}$ were observed to be symmetric, all the parameters were measured on the right half of the structure (lamina \#1 and \#2).

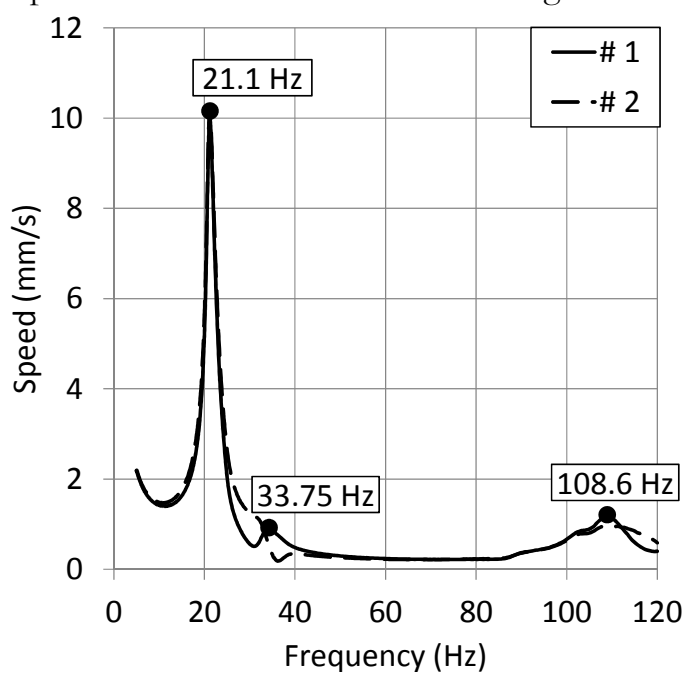

Figure 5: Diagram of the speed registered experimentally on the tip of lamina \#1 (solid line) and lamina \#2 (dashed line).

Fig. 6 describes the tip displacement measured experimentally for each eigenfrequency both for lamina \#1 and \#2 (Fig. 6a and b, respectively) at a base acceleration of 1g. Each bar chart displays three columns for each eigenfrequencies: a solid black, a solid white, and a solid grey column for the resistive load equal to $6.8 \mathrm{M} \Omega$, to $100 \mathrm{k} \Omega$, and to $10 \mathrm{k} \Omega$, respectively. Keeping the same layout, Fig. 7 presents the output root mean square (RMS) voltage.

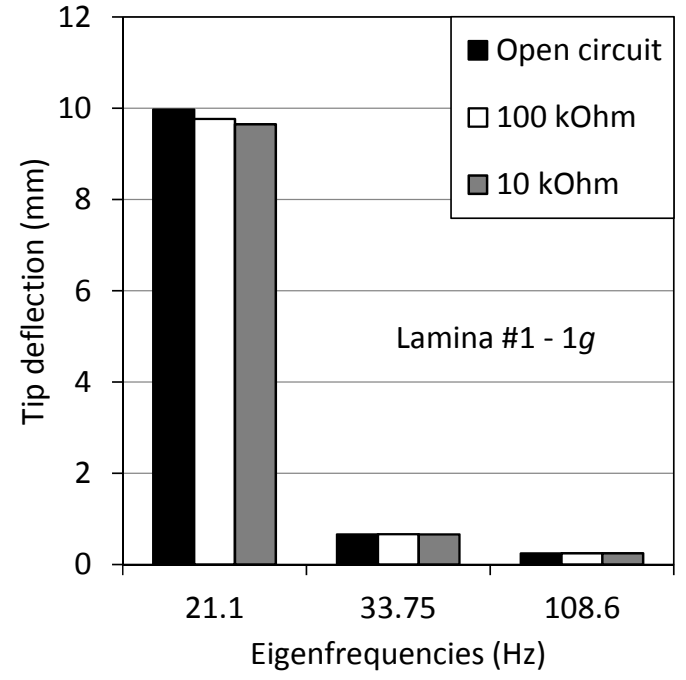

(a)

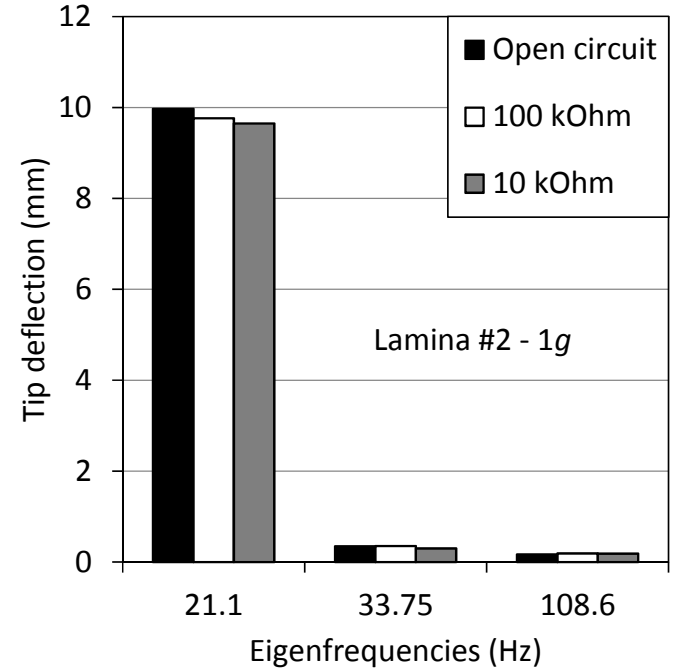

(b)

Figure 6: Bar charts of the tip displacement measured experimentally for each eigenfrequency to an acceleration of $1 \mathrm{~g}$ : lamina \#1 (a), and lamina \#2 (b).

Finally, Fig. 8 shows the bar charts of the total power output generated by the converter at each eigenfrequency, both for an acceleration of $0.5 \mathrm{~g}$ and $1 \mathrm{~g}$ (Fig. $8 \mathrm{a}$ and b, respectively). The power output was calculated according to the following relationship: 


$$
P=\sum_{i=1}^{4} \frac{V_{R M S, i}^{2}}{R}
$$

where $V_{R M S, i}$ is the output root mean square voltage of the $i$-th lamina, and $\mathrm{R}$ is the resistive load applied to each piezoelectric lamina.

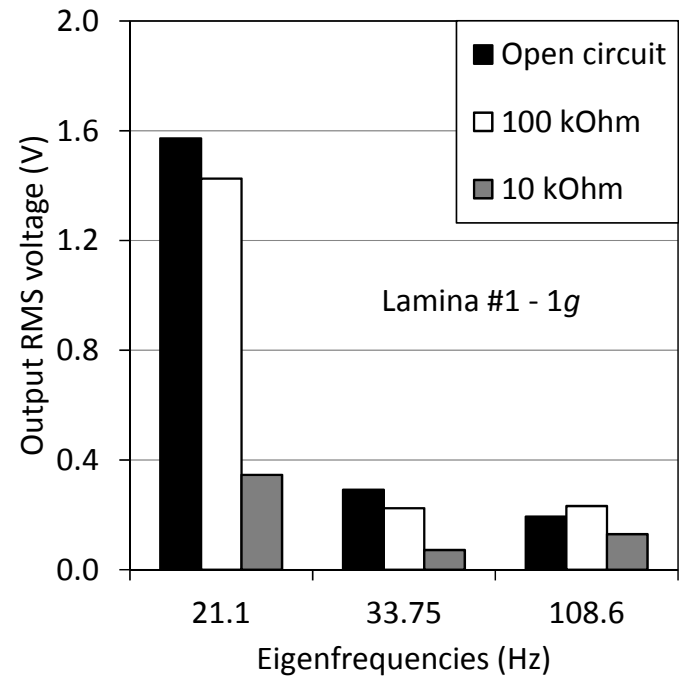

(a)

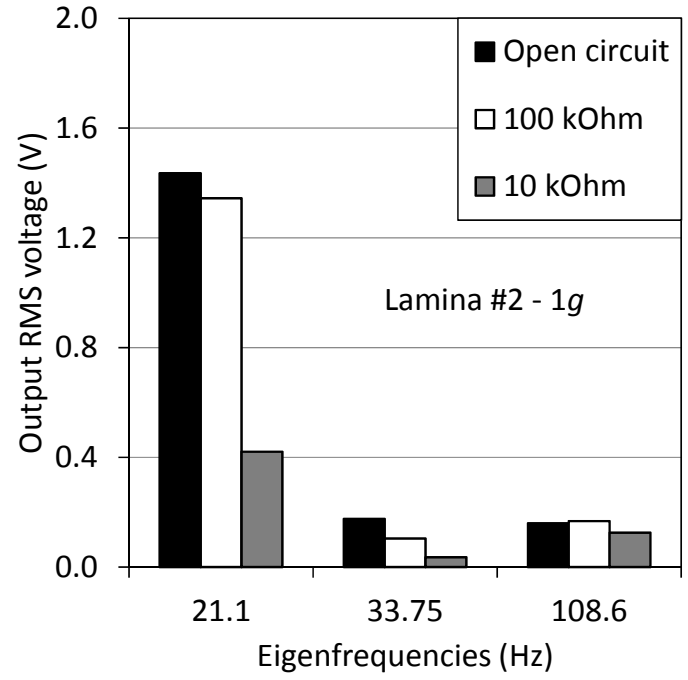

(b)

Figure 7: Bar charts of the output root mean square voltage measured experimentally for each eigenfrequency to an acceleration of $1 \mathrm{~g}$ : lamina \#1 (a), and lamina \#2 (b).

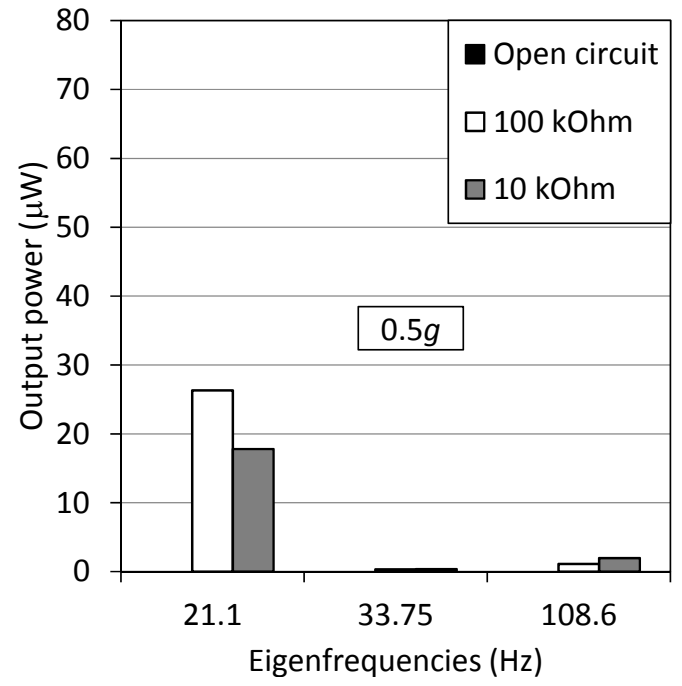

(a)

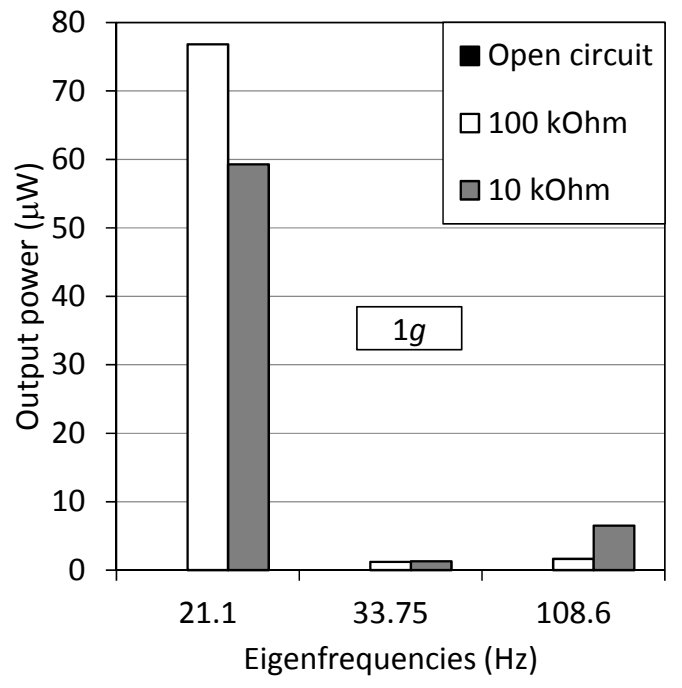

(b)

Figure 8: Bar charts of the total output power measured experimentally for each eigenfrequency under different resistive loads, both at $0.5 \mathrm{~g}$ (a) and $1 \mathrm{~g}$ (b).

\section{Discussion}

$\mathrm{F}$ igure 5 highlights three eigenfrequencies below $120 \mathrm{~Hz}$. The first eigenfrequency involves the whole structure (equal tip speed at $21.1 \mathrm{~Hz}$ both for lamina \#1 and \#2), being the eigenmode of a cantilever structure with the same global shape. The second eigenfrequency $(32.75 \mathrm{~Hz})$ belongs only to lamina \#1 (and its symmetrical \#4). The third eigenfrequency $(108.6 \mathrm{~Hz})$ is again common to the whole structure. 
From Fig. 6 it appears that, at the first eigenfrequency, the tip deflection is exactly the same for all the laminas (as discussed above), and is many times higher than that of subsequent eigenmodes. The resistive load seems to have no effect on the tip deflection. By contrast, despite not showed here for brevity, it was observed that the tip deflection linearly depends on the base acceleration.

The RMS voltage in Fig. 7 is quite similar for lamina \#1 and \#2, and, as expected, the higher values are obtained by increasing the resistive load up to an open circuit condition (solid black bars). A small difference is observed at the third eigenfrequency, where the higher RMS voltage is obtained for a resistive load of $100 \mathrm{k} \Omega$ (empty bars). Probably, a more accurate investigation on this eigenfrequency would be needed. Moreover, the RMS voltage is noticeably low at the first and second eigenfrequency when the $10 \mathrm{k} \Omega$ resistive load (grey bars) is applied to the piezoelectric patches. Similarly to the tip displacement in Fig. 6, also the RMS voltage is much more high at the first eigenfrequency and is proportional to the base acceleration.

Fig. 8 shows that the overall output power of the converter at the fundamental eigenfrequency is an order of magnitude higher than at the second and third eigenfrequencies. The output power increases more than linearly with the base acceleration. In addition, both for the first and third eigenfrequency the resistive load significantly affects power generation. Despite the highest power generation is obtained at the first eigenfrequency, also at subsequent eigenfrequencies the converter is able to provide a useful power output, which can be maximized by choosing the optimal resistive load for that given frequency.

On the whole, a multi-frequency converter allows to harvest ambient energy in a given frequency range and can be particularly efficient in applications dealing with excitations which are not controllable or are intrinsically frequencyvariant over a wide range. Further experimental tests will be performed to compare this fractal-inspired multi-frequency converter with a traditional multi-cantilever solution.

\section{CONCLUSIONS}

A fractal-inspired, multi-frequency, piezoelectric energy converter, which is a square thin sheet structure with inner cuts, is designed and experimentally investigated. The converter exhibits three eigenfrequencies in the range between 0 and $120 \mathrm{~Hz}$ : the first eigenfrequency corresponds to that of an equivalent cantilever, the second and third eigenfrequency comes from the inner cantilevers. The electric power generation under different levels of resistive loads (from open circuit up to a low electric resistance) is good, in particular at the first eigenfrequency, and increases almost linearly with the base acceleration. A multi-frequency converter, as presented here, can be particularly efficient for energy harvesting involving ambient vibrations whose frequency is uncertain or varying over a wide range.

\section{REFERENCES}

[1] G. Despesse, T. Jager, J.J. Chaillout, et al., In: Proc. Ph.D. Res. Microelectron. Electron., 1 (2005) 225.

[2] S.P. Beeby, M.J. Tudor, N.M. White, Meas. Sci. Technol. 17 (2006) R175.

[3] J. Dewei, L. Jing LIU, Front. Energy Power Eng. China, 3(1) (2009) 27.

[4] G. De Pasquale, A. Somà, N. Zampieri, J. of Fuel Cell Science and Technol., 9(4) (2012) 041011.

[5] G. De Pasquale, A. Somà, In: Symposium on Design, Test, Integration and Packaging of MEMS/MOEMS, DTIP (2010) 134.

[6] G. De Pasquale, E. Brusa, A. Somà, In: DTIP of MEMS and MOEMS - Symposium on Design, Test, Integration and Packaging of MEMS/MOEMS, (2009) 280.

[7] V.R. Challa, M. G. Prasad, F.T. Fisher, Smart Mater. Struct., 18 (2009) 095029.

[8] A. Khaligh, P. Zeng, X. Wu, and Y. Xu, In: IECON 2008. 34th Annual Conference of IEEE (2008) 448.

[9] D. Zakharov, G. Lebedev, O. Cugat, J. Delamare, B. Viala, T. Lafont, L. Gimeno, A. Shelyakov, J. Micromech. Microeng., 22 (2012) 094005.

[10] S. R. Anton, A. Erturk, D. J. Inman, Smart Mater. Struct. 19 (2010) 115021.

[11] L. Gu, Microelectronics Journal, 42 (2011) 277.

[12] F. Glynne-Jones, S.P. Beeby, N.M. White, IEE Proc. Sci. Mem. Technol., 148(2) (2001) 68.

[13] S. Zurn, M. Hsieh, G. Smith et al., Smart Mater. Struct. 10 (2001) 252.

[14] S. Roundy, P.K. Wright, J. Rabaey, Computer Communications 26 (2003) 1131.

[15] A. Erturk, D.J. Inman, Smart Mater. Struct., 18 (2009) 1. 
[16] D. Shen, S.Y. Choe, D.J. Kim, Jap. J. Appl. Phys., 46(10) (2007) 6755.

[17] D. Benasciutti, L. Moro, S. Zelenika, and E. Brusa, Microsyst. Technol., 16 (2010) 657.

[18] H.J. Song, Y.T. Choi, G. Wang, et al., J. Mech. Des. 131(9) (2009) 091008.

[19] M. Ferrari, V. Ferrari, M. Guizzetti, et al., Sens. Actuators, 142 (2008) 329.

[20] S. Qi, R. Shuttleworth, S.O. Oyadiji, In: Proceedings of SMASIS, CA, 2009.

[21] S.M. Shahruz, Mechatronics, 16 (2006) 523.

[22] D. Castagnetti, J. of Mech. Design, 133(11) (2011) 111005-1.

[23] D. Castagnetti, In: Proceedings of SMASIS (2011) Arizona.

[24] Piezo System, Inc., USA, www.piezo.com.

[25] http://www.dataphysics.com/

[26] TDS "Miniature Accelerometers" on www.mmf.de.

[27] http://www.polytec.com/us/

[28] http://www.ni.com/products/

[29] http://www.ni.com/labview/ 